\title{
Perturbative quantization of Yang-Mills theory with classical double as gauge algebra
}

\author{
F. Ruiz Ruiz ${ }^{\mathrm{a}}$ \\ Departamento de Física Teórica I, Universidad Complutense de Madrid, 28040 Madrid, Spain
}

Received: 8 December 2015 / Accepted: 8 February 2016 / Published online: 22 February 2016

(C) The Author(s) 2016. This article is published with open access at Springerlink.com

\begin{abstract}
Perturbative quantization of Yang-Mills theory with a gauge algebra given by the classical double of a semisimple Lie algebra is considered. The classical double of a real Lie algebra is a nonsemisimple real Lie algebra that admits a nonpositive definite invariant metric, the indefiniteness of the metric suggesting an apparent lack of unitarity. It is shown that the theory is UV divergent at one loop and that there are no radiative corrections at higher loops. Oneloop UV divergences are removed through renormalization of the coupling constant, thus introducing a renormalization scale. The terms in the classical action that would spoil unitarity are proved to be cohomologically trivial with respect to the Slavnov-Taylor operator that controls gauge invariance for the quantum theory. Hence they do not contribute gauge invariant radiative corrections to the quantum effective action and the theory is unitary.
\end{abstract}

\section{Introduction}

Nonreductive metric Lie algebras are Lie algebras that (i) cannot be written as a direct product of semisimple and Abelian Lie algebras but (ii) admit a metric, where by a metric is meant a nondegenerate symmetric bilinear form that is invariant under the adjoint action. Here we will be concerned with perturbative quantization of Yang-Mills theory for a particular class of such algebras, known as classical doubles. These algebras describe the gauge symmetries in a variety of problems, including three-dimensional gravity [1,2], asymptotically flat solutions to the Einstein equations in three and four dimensions [3-5], string actions in doubled space [6], or $1 / N_{\text {color }}$ expansions for baryons in QCD [7].

Many WZW models [8-14] based on nonreductive Lie algebras, though of a different type, have a simpler structure than the WZW models based on semisimple Lie algebras. This suggests considering Yang-Mills theories in four

a e-mail: ferruiz@ucm.es dimensions and investigate if the simplifications introduced in two dimensions by going nonreductive carry through to four dimensions. The problem was addressed in Ref. [15] for a class of nonreductive algebras called double extensions. One-loop radiative corrections for certain models were computed and it was argued that, if renormalizability is assumed, there would not be higher-loop corrections. An apparent lack of unitarity was found.

The classical double, we denote it as $\mathfrak{g}_{\ltimes}$, of any real Lie algebra $\mathfrak{g}$ is a Lie algebra of dimension twice the dimension of $\mathfrak{g}$ that admits a metric. This metric determines a YangMills Lagrangian for the field that results from gauging the algebra. For $\mathfrak{g}$ simple, the self-antiself dual instantons of the $\mathfrak{g}_{\ltimes}$ Yang-Mills theory in four-dimensional Euclidean space have been studied elsewhere [16]. Every $\mathfrak{g}_{\ltimes}$ instanton has an embedded $\mathfrak{g}$ instanton with the same instanton number and twice the number of collective coordinates. This doubling of degrees of freedom and the simpler structure of classical doubles as compared to double extensions suggest considering perturbative quantization of Yang-Mills theory with gauge algebra $\mathfrak{g}_{\ltimes}$.

The theory is shown to have UV divergences at one loop but no radiative corrections at higher loops. As in the classical case, the physical degrees of freedom of the quantum theory are doubled with respect to the $\mathfrak{g}$ theory. In particular, the first and only coefficient (since there are no radiative corrections beyond one loop) of the beta function is twice that of the $\mathfrak{g}$ theory. To disentangle truly gauge invariant one-loop corrections from those due to gauge fixing, the SlavnovTaylor operator for the quantum theory is used. The term in the classical action that would spoil unitarity is cohomologically trivial with respect to the Slavnov-Taylor operator, so in the quantum effective action it can be put to zero through a field redefinition and poses no problem for unitarity.

The manuscript is organized as follows. Section 2 contains a brief reminder of classical doubles $\mathfrak{g}_{\ltimes}$ and their Lie groups $G_{\ltimes}$. Classical Yang-Mills theory with gauge 
group $G_{\ltimes}$ is formulated in Sect. 3, and the path integral generating the theory's Green functions is derived. With the mind set in avoiding miscounting the theory's degrees of freedom, special consideration is given to gauge fixing, and three derivations for the gauge fixing terms in Landau gauge are presented. Section 3 also discusses the emergence of the classical theory as a limit of Yang-Mills theory with gauge algebra the direct product $\mathfrak{g} \times \mathfrak{g}$. Section 4 contains our perturbative analysis, with the calculation of one-loop 1PI radiative corrections and the proof that there are no higher-loop corrections. The one-loop divergences are removed in Sect. 5 by adding a gauge invariant counterterm consistent with unitarity, so the one-loop contribution to the quantum effective action is positive definite. We conclude in Sect. 6.

\section{Semidirect products of Lie algebras and their groups}

We start by recalling the definitions of classical double of a Lie algebra and its Lie group. Consider an arbitrary Lie algebra $\mathfrak{g}$ of dimension $n$ with basis $\left\{T_{a}\right\}$ and commutation relations $\left[T_{a}, T_{b}\right]=f_{a b}{ }^{c} T_{c}$. As a vector space, $\mathfrak{g}$ has a dual vector space $\mathfrak{g}^{*}$. Take on $\mathfrak{g}^{*}$ the canonical dual basis $\left\{Z^{a}\right\}$, given by $Z^{a}\left(T_{b}\right)=\delta^{a}{ }_{b}$. The classical double of $\mathfrak{g}$, denoted by $\mathfrak{g}_{\ltimes}$, is the Lie algebra of dimension $2 n$ with basis $\left\{T_{a}, Z^{b}\right\}$ and commutators

$\left[T_{a}, T_{b}\right]=f_{a b}^{c} T_{c}, \quad\left[T_{a}, Z^{b}\right]=-f_{a c}^{b} Z^{c},\left[Z^{a}, Z^{b}\right]=0$.

It is trivial to check that these commutators satisfy the Jacobi identity, so indeed they define a Lie bracket. In a more mathematical language [14], the algebra $\mathfrak{g}_{\ltimes}$ is the semidirect product $\mathfrak{g} \ltimes \mathfrak{g}^{*}$ obtained by the coadjoint action of $\mathfrak{g}$ on $\mathfrak{g}^{*}$.

It is clear from the commutators (2.1) that $\mathfrak{g}_{\ltimes}$ is not semisimple, so its Killing form is degenerate and cannot be used as a metric. For convenience we recall that a metric on a Lie algebra is a symmetric, nondegenerate, bilinear form $\Omega$ such that

$\Omega(A,[B, C])=\Omega([A, B], C)$

for all $A, B, C$ in the algebra. The relevance of condition (2.2) is that it implies invariance under the adjoint action of the algebra's Lie group,

$\Omega\left(e^{-C} A e^{C}, e^{-C} B e^{C}\right)=\Omega(A, B)$.

It is straightforward to check that, for $\left\{T_{a}, Z^{b}\right\}$ above, the bilinear symmetric form

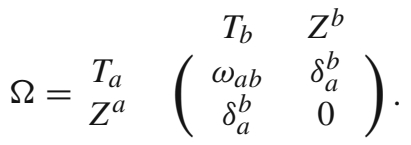

is nondegenerate and solves Eq. (2.2), hence is a metric on $\mathfrak{g}_{\ltimes}$. Here $\omega_{a b}=\omega\left(T_{a}, T_{b}\right)$ is an arbitrary invariant, symmetric, bilinear form on $\mathfrak{g}$ that may be degenerate. It is worth remarking that $\mathfrak{g}$ is arbitrary and need not be metric itself.

Denote by $\mathrm{G}_{\ltimes}, \mathrm{G}$, and $\mathrm{N}$ the Lie groups obtained by exponentiation of the algebras $\mathfrak{g}_{\ltimes}, \mathfrak{g}$, and $\mathfrak{g}^{*}$. Every element $h$ of $\mathrm{G}$ and every $n$ of $\mathrm{N}$ can be uniquely written as $h=e^{T}$ and $n=e^{Z}$, for some $T$ in $\mathfrak{g}$ and some $Z$ in $\mathfrak{g}^{*}$. In turn, every element $g$ of $\mathrm{G}_{\ltimes}$ can be uniquely written as $g=h n$. The product $g_{3}=g_{1} g_{2}$ of two elements $g_{1}=h_{1} n_{1}$ and $g_{2}=h_{2} n_{2}$ of $\mathrm{G}_{\ltimes}$ is given by $g_{3}=h_{3} n_{3}$, with $h_{3}=h_{1} h_{2}$ and $n_{3}=\left(h_{2}^{-1} n_{1} h_{2}\right) n_{2}$. It is clear that $g_{3}$ is in $\mathrm{G}_{\ltimes}$ since the Campbell-Hausdorff formula and the commutators (2.1) imply that $h_{3}$ is in G and $n_{3}$ is in N. It is very easy to see that the group $G_{\ltimes}$ is the semidirect product of $G$ with the normal Abelian subgroup N. Since N is isomorphic to $\mathbf{R}^{n}$, the group $\mathrm{G}_{\ltimes}$ is noncompact.

So far no restriction has been placed on $\mathfrak{g}$. Assume now that it is semisimple. In this case, $\mathfrak{g}_{\ltimes}$ can be viewed as a limit of the direct product of $\mathfrak{g}$ with itself [16]. To see this, take $\omega_{a b}$ in Eq. (2.4) proportional to the Killing form of $\mathfrak{g}$. Since $\omega_{a b}$ is nondegenerate, indices in the structure constants $f_{a b}{ }^{c}$ can be raised and lowered using $\omega_{a b}$ and its inverse $\omega^{a b}$, defined by $\omega^{a b} \omega_{b c}=\delta^{a}{ }_{c}$. This gives completely antisymmetric structure constants,

$f_{a b c}=f_{a b}{ }^{d} \omega_{d c}, \quad f_{a b c}=-f_{b a c}=f_{b c a}$.

Perform in $\mathfrak{g}^{*}$ the change of generators $Z^{a} \rightarrow Z_{a}=\omega_{a b} Z^{b}$. In the basis $\left\{T_{a}, Z_{b}\right\}$ the Lie bracket (2.1) reads

$$
\left[T_{a}, T_{b}\right]=f_{a b}^{c} T_{c}, \quad\left[T_{a}, Z_{b}\right]=f_{a b}^{c} Z_{c}, \quad\left[Z_{a}, Z_{b}\right]=0
$$

and the metric $\Omega$ is recast as

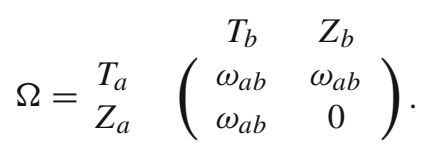

Consider now the commutators

$$
\begin{gathered}
{\left[T_{a}, T_{b}\right]=f_{a b}{ }^{c} T_{c}, \quad\left[T_{a}, Z_{b}\right]=f_{a b}^{c} Z_{c},} \\
{\left[Z_{a}, Z_{b}\right]=t^{2} f_{a b}{ }^{c} T_{c},}
\end{gathered}
$$

where $t$ is an arbitrary real parameter. It is trivial to show that they satisfy the Jacobi identity for all $t$. Hence they define a Lie algebra, call it $\mathfrak{g}_{\ltimes}^{t}$, that reduces to $\mathfrak{g}_{\ltimes}$ in the limit $t \rightarrow 0$. Furthermore, $\mathfrak{g}_{\ltimes}^{t}$ admits the invariant metric 


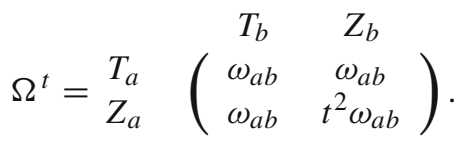

The change of basis

$X_{a}^{ \pm}=\frac{1}{2}\left(T_{a} \pm \frac{1}{t} Z_{a}\right)$,

in $\mathfrak{g}_{\ltimes}^{t}$ transforms the Lie bracket (2.7) in

$\left[X_{a}^{ \pm}, X_{b}^{ \pm}\right]=f_{a b}^{c} X_{c}^{ \pm}, \quad\left[X_{a}^{+}, X_{b}^{-}\right]=0$

and gives for the metric $\Omega^{t}$ in Eq. (2.8) the block diagonal form

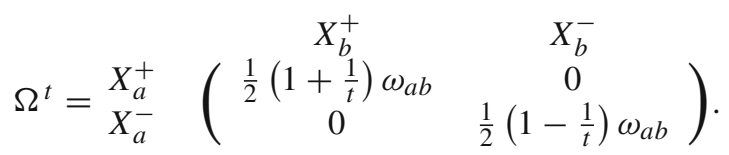

It is clear from Eqs. (2.10) and (2.11) that $\mathfrak{g}_{\times}^{t}$ is the direct product $\mathfrak{g} \times \mathfrak{g}$, so its Lie group $\mathrm{G}_{\ltimes}^{t}$ is the direct product $\mathrm{G} \times \mathrm{G}$.

\section{The gauge fixed classical action}

We assume from now on that $\mathfrak{g}$ is semisimple and work in the basis $\left\{T_{a}, Z_{b}\right\}$ of $\mathfrak{g}_{\ltimes}$. The commutation relations are as in Eq. (2.5) and the metric $\Omega$ as in (2.6). The Lie groups of $\mathfrak{g}$ and $\mathfrak{g}_{\ltimes}$ will be denoted by $G$ and $G_{\ltimes}$. All quantities taking values in $\mathfrak{g}_{\ltimes}$ will be written in boldface, whereas their $T_{a}$ and $Z_{a}$ components will be labeled with subscripts $\mathrm{T}$ and $\mathrm{Z}$. For the gauge field $\boldsymbol{A}_{\mu}$ and its field strength $\boldsymbol{F}_{\mu \nu}=\partial_{\mu} \boldsymbol{A}_{\nu}$ $\partial_{\nu} \boldsymbol{A}_{\mu}+\left[\boldsymbol{A}_{\mu}, \boldsymbol{A}_{\nu}\right]$, we have

$$
\begin{aligned}
\boldsymbol{A}_{\mu} & =A_{\mathrm{T} \mu}^{a} T_{a}+A_{\mathrm{Z} \mu}^{a} Z_{a}, \\
\boldsymbol{F}_{\mu \nu} & =F_{\mathrm{T} \mu \nu}^{a} T_{a}+F_{\mathrm{Z} \mu \nu}^{a} Z_{a} .
\end{aligned}
$$

The expressions of $F_{\mathrm{T} \mu \nu}^{a}$ and $F_{\mathrm{Z} \mu \nu}^{a}$ in terms of $A_{\mathrm{T} \mu}^{a}$ and $A_{\mathrm{Z} \mu}^{a}$ follow from the commutators (2.5),

$$
\begin{aligned}
& F_{\mathrm{T} \mu \nu}^{a}=\partial_{\mu} A_{\mathrm{T} \nu}^{a}-\partial_{\nu} A_{\mathrm{T} \mu}^{a}+f_{b c}{ }^{a} A_{\mathrm{T} \mu}^{b} A_{\mathrm{Z} \nu}^{c}, \\
& F_{\mathrm{Z} \mu \nu}^{a}=\partial_{\mu} A_{\mathrm{Z} \nu}^{a}-\partial_{\nu} A_{\mathrm{Z} \mu}^{a}+f_{b c}{ }^{a}\left(A_{\mathrm{T} \mu}^{b} A_{\mathrm{Z} \nu}^{a}-A_{\mathrm{T} \nu}^{b} A_{\mathrm{Z} \nu}^{a}\right) .
\end{aligned}
$$

The action of the covariant derivative $\boldsymbol{D}_{\mu}=\partial_{\mu}+\left[\boldsymbol{A}_{\mu}\right.$, $]$ on any tensor $\boldsymbol{\Phi}=\Phi_{\mathrm{T}}^{a} T_{a}+\Phi_{\mathrm{Z}}^{a} Z_{a}$ has components

$$
\begin{aligned}
& \left(\boldsymbol{D}_{\mu} \boldsymbol{\Phi}\right)_{\mathrm{T}}^{a}=\partial_{\mu} \Phi_{\mathrm{T}}^{a}+f_{b c}{ }^{a} A_{\mathrm{T} \mu}^{b} \Phi_{\mathrm{T}}^{c}, \\
& \left(\boldsymbol{D}_{\mu} \boldsymbol{\Phi}\right)_{\mathrm{Z}}^{a}=\partial_{\mu} \Phi_{\mathrm{Z}}^{a}+f_{b c}{ }^{a} A_{\mathrm{T} \mu}^{b} \Phi_{\mathrm{Z}}^{c}+f_{b c}{ }^{a} A_{\mathrm{Z} \mu}^{b} \Phi_{\mathrm{T}}^{c} .
\end{aligned}
$$

On the right hand side of the first equation, one recognizes the covariant derivative for the algebra $\mathfrak{g}$, which we denote by $D_{\mu}$,

$$
D_{c}^{a_{\mu}}:=\delta_{c}{ }^{a} \partial_{\mu}+f_{b c}{ }^{a} A_{\mathrm{T} \mu}^{b} \text {. }
$$

Finite gauge transformations read

$$
\begin{aligned}
& \boldsymbol{A}_{\mu} \rightarrow \boldsymbol{A}_{\mu}^{\prime}=g^{-1} \partial_{\mu} g+g^{-1} \boldsymbol{A}_{\mu} g, \\
& \boldsymbol{F}_{\mu \nu} \rightarrow \boldsymbol{F}_{\mu \nu}^{\prime}=g^{-1} \boldsymbol{F}_{\mu \nu} g,
\end{aligned}
$$

with $g$ an arbitrary group element in $\mathrm{G}_{\ltimes}$,

$g(x)=\exp \left[\theta_{\mathrm{T}}^{a}(x) T_{a}\right] \exp \left[\theta_{\mathrm{Z}}^{a}(x) Z_{a}\right]$.

Infinitesimally these transformations take the form

$\delta A_{\mathrm{T} \mu}^{a}=D_{\mu} \theta_{\mathrm{T}}^{a}$,
$\delta A_{\mathrm{Z} \mu}^{a}=D_{\mu} \theta_{\mathrm{Z}}^{a}+f_{b c}{ }^{a} A_{\mathrm{Z} \mu}^{b} \theta_{\mathrm{T}}^{c}$

for the gauge field, and

$\delta F_{\mathrm{T} \mu \nu}^{a}=f_{b c}{ }^{a} F_{\mathrm{T} \mu \nu}^{b} \theta_{\mathrm{T}}^{c}$,

$\delta F_{\mathrm{Z} \mu \nu}^{a}=f_{b c}{ }^{a}\left(F_{\mathrm{Z} \mu \nu}^{b} \theta_{\mathrm{T}}^{c}+F_{\mathrm{T} \mu \nu}^{b} \theta_{\mathrm{Z}}^{c}\right)$

for the field strength. The invariance condition (2.3) for the metric $\Omega$ and the transformation law (3.2) for the field strength $\boldsymbol{F}_{\mu \nu}$ imply that the Lagrangian density

$$
\begin{aligned}
\mathcal{L}_{\mathrm{YM}} & =\frac{1}{4 g^{2}} \Omega\left(\boldsymbol{F}_{\mu \nu}, \boldsymbol{F}^{\mu \nu}\right) \\
& =\frac{1}{4 g^{2}} \omega_{a b}\left(F_{\mathrm{T} \mu \nu}^{a} F_{\mathrm{T}}^{b \mu \nu}+2 F_{\mathrm{T} \mu \nu}^{a} F_{\mathrm{Z}}^{b \mu \nu}\right),
\end{aligned}
$$

where $g$ is a coupling constant, is gauge invariant. We are interested in perturbatively quantizing Yang-Mills theory with Lagrangian $\mathcal{L}_{\mathrm{YM}}$. To obtain a path integral that generates the theory's Green functions, we next fix the gauge. We do this in three different ways.

Gauge fixing I. Introduce a ghost field $\boldsymbol{c}$, an antighost field $\overline{\boldsymbol{c}}$ and a Lagrange multiplier field $\boldsymbol{b}$,

$\boldsymbol{c}=c_{\mathrm{T}}^{a} T_{a}+c_{\mathrm{Z}}^{a} Z_{a}$,

$\overline{\boldsymbol{c}}=\bar{c}_{\mathrm{T}}^{a} T_{a}+\bar{c}_{\mathrm{Z}}^{a} Z_{a}$,

$\boldsymbol{b}=b_{\mathrm{T}}^{a} T_{a}+b_{\mathrm{Z}}^{a} Z_{a}$.

Use the infinitesimal form of the gauge transformations to define a BRS operator $s$ by its action on the fields,

$s \boldsymbol{A}_{\mu}=\boldsymbol{D}_{\mu} \boldsymbol{c}, \quad s \boldsymbol{c}=-\boldsymbol{c c}, \quad s \overline{\boldsymbol{c}}=\boldsymbol{b}, \quad s \boldsymbol{b}=0$.

The operator $s$ commutes with $\partial_{\mu}, \boldsymbol{A}_{\mu}$ and $\boldsymbol{b}$, and anticommutes with $\boldsymbol{c}$ and $\bar{c}$. The BRS transformations for the $\mathrm{T}$ and $\mathrm{Z}$ components of the fields can be obtained either from the definition of $s$ in Eq. (3.8) and the commutations relations (2.5), or directly from Eqs. (3.3) and (3.4). They read

$$
\begin{aligned}
s A_{\mathrm{T} \mu} & =D_{\mu} c_{\mathrm{T}}^{a}, & & s c_{\mathrm{T}}^{a}=-\frac{1}{2} f_{b c}{ }^{a} c_{\mathrm{T}}^{b} c_{\mathrm{T}}^{c}, \\
s \bar{c}_{\mathrm{T}}^{a} & =b_{\mathrm{T}}^{a}, & & s b_{\mathrm{T}}^{a}=0,
\end{aligned}
$$


and

$$
\begin{aligned}
& s A_{\mathrm{Z} \mu}^{a}=D_{\mu} c_{\mathrm{Z}}^{a}+f_{b c}{ }^{a} A_{\mathrm{Z} \mu}^{b} c_{\mathrm{T}}^{c}, \\
& \quad s c_{\mathrm{Z}}^{a}=-f_{b c}{ }^{a} c_{\mathrm{T}}^{b} c_{\mathrm{Z}}^{c}, s \bar{c}_{\mathrm{Z}}^{a}=b_{\mathrm{Z}}^{a}, s b_{\mathrm{Z}}^{a}=0 .
\end{aligned}
$$

Using Eqs. (3.8), or their equivalent Eqs. (3.9) and (3.10), it is very easy to check that $s^{2}=0$. Note that the BRS transformations (3.9) are the same as for the semisimple gauge algebra $\mathfrak{g}$. In Lorenz gauge, the gauge fixing Lagrangian is the BRS variation

$\mathcal{L}_{\mathrm{GF}}=-\frac{1}{g^{2}} s \Omega\left(\overline{\boldsymbol{c}}, \frac{\alpha}{2} \boldsymbol{b}+\partial \boldsymbol{A}\right)$,

where $\alpha$ is the gauge parameter and a contraction of the spacetime indices in $\partial_{\mu}$ and $\boldsymbol{A}_{\mu}$ is understood. Expanding in terms of field components, $\mathcal{L}_{\mathrm{GF}}$ becomes

$$
\begin{aligned}
\mathcal{L}_{\mathrm{GF}}=\frac{1}{g^{2}} \omega_{a b}[ & -\frac{\alpha}{2}\left(b_{\mathrm{T}}^{a} b_{\mathrm{T}}^{b}+2 b_{\mathrm{T}}^{a} b_{\mathrm{Z}}^{b}\right)-b_{\mathrm{T}}^{a}\left(\partial A_{\mathrm{T}}^{b}\right) \\
& -b_{\mathrm{T}}^{a}\left(\partial A_{\mathrm{Z}}^{b}\right)-b_{\mathrm{Z}}^{a}\left(\partial A_{\mathrm{T}}^{b}\right) \\
& +\bar{c}_{\mathrm{T}}^{a}\left(\partial D c_{\mathrm{T}}^{b}\right)+\bar{c}_{\mathrm{T}}^{a}\left(\partial D c_{\mathrm{Z}}^{b}\right) \\
& \left.+\bar{c}_{\mathrm{T}}^{a} \partial\left(f_{c d}{ }^{b} A_{\mathrm{Z}}^{c} c_{\mathrm{T}}^{d}\right)+\bar{c}_{\mathrm{Z}}^{a}\left(\partial D c_{\mathrm{T}}^{b}\right)\right],
\end{aligned}
$$

with $D_{\mu}$ the $\mathfrak{g}$ covariant derivative in Eq. (3.1). The gauge fixed Lagrangian is the sum

$\mathcal{L}_{\ltimes}=\mathcal{L}_{\mathrm{YM}}+\mathcal{L}_{\mathrm{GF}}$.

Introduce external sources $\left\{\boldsymbol{J}_{\boldsymbol{\Phi}}\right\}=\left\{\boldsymbol{J}^{\mu}, \overline{\boldsymbol{\zeta}}, \boldsymbol{\zeta}, \boldsymbol{B}\right\}$ for the fields $\{\boldsymbol{\Phi}\}=\left\{\boldsymbol{A}_{\mu}, \boldsymbol{c}, \overline{\boldsymbol{c}}, \boldsymbol{b}\right\}$, with

$J_{\Phi}=J_{\Phi}^{a} T_{a}+J_{\Phi \mathrm{Z}}^{a} Z_{a}$.

The path integral that generates the theory's Green functions is given by

$$
\begin{aligned}
Z[\boldsymbol{J}, \bar{\zeta}, \zeta, \boldsymbol{B}]= & \int[\mathrm{d} \boldsymbol{A}][\mathrm{d} \boldsymbol{c}][\mathrm{d} \overline{\boldsymbol{c}}][\mathrm{d} \boldsymbol{b}] \\
& \exp \left[-\int \mathrm{d}^{4} x \mathcal{L}_{\ltimes}+S_{\text {ext }}\right],
\end{aligned}
$$

where $[\mathrm{d} \boldsymbol{\Phi}]=\left[\mathrm{d} \Phi_{\mathrm{T}}\right]\left[\mathrm{d} \Phi_{\mathrm{Z}}\right]$ for every field $\boldsymbol{\Phi}$, and $S_{\text {ext }}$ is the source term

$$
\begin{aligned}
S_{\mathrm{ext}}= & \frac{1}{g^{2}} \int \mathrm{d}^{4} x[\Omega(\boldsymbol{J}, \boldsymbol{A})+\Omega(\bar{\zeta}, \boldsymbol{c})+\Omega(\overline{\boldsymbol{c}}, \zeta) \\
& +\Omega(\boldsymbol{B}, \boldsymbol{b})] .
\end{aligned}
$$

The external sources are coupled to fields through the Lie algebra metric $\Omega$, so that

$$
\Omega\left(\boldsymbol{J}_{\boldsymbol{\Phi}}, \boldsymbol{\Phi}\right)=\omega_{a b}\left(J_{\Phi_{\mathrm{T}}^{a}}^{a} \Phi_{\mathrm{T}}^{b}+J_{\Phi}^{a} \Phi_{\mathrm{Z}}^{b}+J_{\Phi}{ }_{\mathrm{Z}}^{a} \Phi_{\mathrm{T}}^{b}\right)
$$

for every field $\boldsymbol{\Phi}$ and its source $\boldsymbol{J}_{\boldsymbol{\Phi}}$. The Green functions are obtained by functionally differentiating with respect to the external sources. For example, $\left\langle A_{\mathrm{T} \mu}^{a}(x) A_{\mathrm{Z} v}^{b}(y)\right\rangle$ is given by $\left.\omega^{a c} \frac{\delta}{\delta J_{\mathrm{Z}}^{c \mu}(x)} \omega^{b d}\left[\frac{\delta}{\delta J_{\mathrm{T}}^{d v}(y)}-\frac{\delta}{\delta J_{\mathrm{Z}}^{d v}(y)}\right] \ln Z\left[\boldsymbol{J}_{\boldsymbol{\phi}}\right]\right|_{\boldsymbol{J}_{\boldsymbol{\phi}}=0}$.

Gauge fixing II. Equivalently, the path integral (3.13) can be derived as follows. In the naive path integral

$\int[\mathrm{d} \boldsymbol{A}] \exp \left[-\int \mathrm{d}^{4} x \mathcal{L}_{\mathrm{YM}}\right]$,

to avoid integrating over gauge equivalent degrees of freedom, replace

$\int[\mathrm{d} \boldsymbol{A}] \rightarrow \int[\mathrm{d} \boldsymbol{A}] \delta(\partial \boldsymbol{A}-\boldsymbol{f}) \Delta_{\partial \boldsymbol{A}}$,

where the Dirac delta imposes the Lorenz gauge fixing condition $\partial A=f$ and

$\Delta_{\partial \boldsymbol{A}}=\operatorname{det}\left[\frac{\delta}{\delta \boldsymbol{\theta}(y)} \partial(\boldsymbol{A}+\delta \boldsymbol{A})(x)\right]_{\partial \boldsymbol{A}=0}$

is the corresponding Faddeev-Popov determinant. Proceed now as usual:

(i) Average over $f$ with Gaussian type weight. That is, introduce in the measure

$$
\int[\mathrm{d} \boldsymbol{f}] \exp \left[-\frac{1}{2 \alpha g^{2}} \int \mathrm{d}^{4} x \Omega(\boldsymbol{f}, \boldsymbol{f})\right] .
$$

(ii) Exponentiate $\delta(\partial \boldsymbol{A}-\boldsymbol{f})$ by means of an auxiliary field b,

$$
\delta(\partial \boldsymbol{A}-\boldsymbol{f})=\int[\mathrm{d} \boldsymbol{b}] \exp \left[\frac{i}{g^{2}} \int \Omega(\boldsymbol{b}, \partial \boldsymbol{A}-\boldsymbol{f})\right] .
$$

(iii) Write the determinant

$$
\Delta_{\partial A}=\operatorname{det} \delta^{(4)}(x-y)\left(\begin{array}{cc}
\partial^{\mu} D_{c}^{a} \mu^{a}(x) & 0 \\
f_{b c}{ }^{a} \partial^{\mu} A_{Z \mu}^{b}(x) & \partial^{\mu} D_{c}^{a}(x)
\end{array}\right)
$$

as a path integral over Grassmann fields $c_{1}^{a}, c_{2}^{a}$, and $\bar{c}_{1 a}, \bar{c}_{2 a}$,

$$
\begin{aligned}
\Delta_{\partial \boldsymbol{A}}= & \int\left[\mathrm{d} \bar{c}_{1}\right]\left[\mathrm{d} \bar{c}_{2}\right]\left[\mathrm{d} c_{1}\right]\left[\mathrm{d} c_{2}\right] \exp \\
& \times\left[-\frac{1}{g^{2}} \int \mathrm{d}^{4} x\left(\bar{c}_{1 a} \partial D c_{1}^{a}+\bar{c}_{2 a} f_{c b}^{a}\right.\right. \\
& \left.\left.\times \partial_{\mu}\left(A_{\mathrm{T}}^{c \mu} c_{1}^{b}\right)+\bar{c}_{2 a} \partial D c_{2}^{a}\right)\right] .
\end{aligned}
$$


Use next (i)-(iii) in Eq. (3.16), integrate over $\boldsymbol{f}$, make the change $^{1}$

$c_{\mathrm{T}}^{a}=c_{1}^{a}, \quad c_{\mathrm{Z}}^{a}=c_{2}^{a}$

$\bar{c}_{\mathrm{T}}^{a}=\omega^{a b} \bar{c}_{2 b}, \quad \bar{c}_{\mathrm{Z}}^{a}=\omega^{a b}\left(\bar{c}_{1 b}-\bar{c}_{2 b}\right)$,

and replace $\boldsymbol{b}$ with $i \boldsymbol{b}$. This gives for the path integral the expression in Eq. (3.13).

Gauge fixing III. The observation at the end of Sect. 2 concerning deformations of $\mathfrak{g}_{\ltimes}$ suggests that classical $G_{\ltimes}$ YangMills theory can be regarded as a limit of $\mathrm{G} \times \mathrm{G}$ Yang-Mills theory. To see this, consider two copies of a Yang-Mills theory, both with gauge group the semisimple Lie group G. Label the copies with the subscripts + and -, so that $A_{ \pm \mu}^{a}, F_{ \pm \mu \nu}^{a}$, and $g_{ \pm}$denote their gauge fields, field strengths and coupling constants. Fix in both copies a Lorenz gauge by introducing ghost, antighost, and auxiliary fields $c_{ \pm}^{a}, \bar{c}_{ \pm}^{a}$, and $b_{ \pm}^{a}$, and a BRS operators $s$ given by

$$
\begin{aligned}
s A_{ \pm \mu}^{a} & =D_{ \pm \mu} c_{ \pm}^{a}, \quad s c_{ \pm}^{a}=-\frac{1}{2} f_{b c}{ }^{a} c_{ \pm}^{b} c_{ \pm}^{c}, \\
s \bar{c}_{ \pm}^{a} & =b_{ \pm}^{a}, \quad s b_{ \pm}^{a}=0 .
\end{aligned}
$$

The corresponding gauge fixed Lagrangians read

$$
\begin{aligned}
\mathcal{L}_{ \pm} & =\frac{1}{4 g_{ \pm}^{2}} \omega_{a b} F_{ \pm \mu \nu}^{a} F_{ \pm}^{b \mu \nu} \\
& -\frac{1}{g_{ \pm}^{2}} \omega_{a b} s\left[\bar{c}_{ \pm}^{a}\left(\frac{\alpha_{ \pm}}{2} b_{ \pm}^{b}+\partial A_{ \pm}^{b}\right)\right] .
\end{aligned}
$$

The sum

$\mathcal{L}_{+-}=\mathcal{L}_{+}+\mathcal{L}_{-}$

describes two theories not interacting with each other. Write $g_{ \pm}$in terms of a coupling constant $g$ and a parameter $t$ as

$\frac{1}{g_{ \pm}^{2}}=\frac{1}{2 g^{2}}\left(1 \pm \frac{1}{t}\right)$

and introduce fields $\Phi_{\mathrm{T}}^{a}$ and $\Phi_{\mathrm{Z}}^{a}$ given by

$\Phi_{\mathrm{T}}^{a}=\frac{1}{2}\left(\Phi_{+}^{a}+\Phi_{-}^{a}\right), \quad \Phi_{\mathrm{Z}}^{a}=\frac{1}{2 t}\left(\Phi_{+}^{a}-\Phi_{-}^{a}\right)$

for $\Phi_{ \pm}^{a}=A_{ \pm \mu}^{a}, c_{ \pm}^{a}, \bar{c}_{ \pm}^{a}, b_{ \pm}^{a}$, Take now $\alpha_{+}=\alpha_{-}=\alpha$ and send $t \rightarrow 0$. In this limit, the + and - sectors couple and the Lagrangian $\mathcal{L}_{+-}$becomes the Lagrangian $\mathcal{L}_{\ltimes}$ of the $\mathrm{G}_{\ltimes}$ theory given by Eqs. (3.7), (3.11) and (3.12).

${ }_{1}$ A more precise notation for the $\mathrm{T}$ and $\mathrm{Z}$ components of a $\mathfrak{g}_{\ltimes}$-valued field is $\boldsymbol{\Phi}=\Phi^{T_{a}} T_{a}+\Phi^{Z_{a}} Z_{a}$. Not to load the writing, we have used instead $\Phi_{\mathrm{T}}^{a}:=\Phi^{T_{a}}$ and $\Phi_{\mathrm{Z}}^{a}:=\Phi^{Z_{a}}$. Using $\Omega$ and $\Omega^{-1}$ to lower and raise indices, one has

$$
\begin{aligned}
\Phi_{T_{a}} & =\omega_{a b}\left(\Phi_{\mathrm{T}}^{b}+\Phi_{\mathrm{Z}}^{b}\right), & \Phi_{Z_{a}} & =\omega_{a b} \Phi_{\mathrm{T}}^{b} \\
\Phi_{\mathrm{T}}^{a} & =\omega^{a b} \phi_{Z_{b}}, & \Phi_{\mathrm{Z}}^{a} & =\omega^{a b}\left(\Phi_{T_{b}}-\Phi_{Z_{b}}\right) .
\end{aligned}
$$

Hence $\left(\bar{c}_{1 a}, \bar{c}_{2 a}\right)$ in Eq. (3.17) is nothing but $\left(\bar{c}_{T_{a}}, \bar{c}_{Z_{a}}\right)$.

\section{Radiative corrections}

To explicitly calculate radiative corrections, we take in this section $\mathfrak{g}$ to be $\mathfrak{s u}(\mathrm{N})$. The group $\mathrm{G}$ is then $\mathrm{SU}(\mathrm{N})$ and for $\mathrm{G}_{\ltimes}$ we use the notation $\mathrm{SU}(\mathrm{N})_{\ltimes}$.

In the conventions of Sect. 2, in which a group element $h$ of SU(N) is written as $e^{T}$, the elements $T$ of $\mathfrak{s u}(N)$ in the defining (fundamental) representation are traceless antihermitean matrices. We normalize the structure constants $f_{a b}{ }^{c}$ of $\mathfrak{s u}(N)$ by requiring $f_{c a}{ }^{d} f_{d b}{ }^{c}=\mathrm{N} \delta_{a b}$. This gives for the Killing form $k_{a b}=\mathrm{N} \delta_{a b}$ and amounts to taking $\operatorname{tr}\left[T_{(R) a} T_{(R) b}\right]=C_{2} \delta_{a b}$ in a representation $R$, with $C_{2}=\mathrm{N}$ in the adjoint representation and $C_{2}=-1 / 2$ in the defining representation. For $\omega_{a b}$ in Eq. (2.6), we take

$\omega_{a b}=\delta_{a b}$,

so indices in $f_{a b}{ }^{c}$ are lowered and raised with $\delta_{a b}$ and $\delta^{a b}$.

Feynman rules in Lorenz gauge. Introduce external sources $\boldsymbol{K}^{\mu}$ and $\boldsymbol{H}$ for the nonlinear BRS transforms $s \boldsymbol{A}_{\mu}$ and $s \boldsymbol{c}$,

$$
\begin{aligned}
& \boldsymbol{K}^{\mu}=K_{\mathrm{T}}^{a \mu} T_{a}+K_{\mathrm{Z}}^{a \mu} Z_{a}, \\
& \boldsymbol{H}^{\mu}=H_{\mathrm{T}}^{a} T_{a}+H_{\mathrm{Z}}^{a} Z_{a} .
\end{aligned}
$$

The path integral (3.13) becomes

$$
\begin{aligned}
& Z[\boldsymbol{J}, \bar{\zeta}, \zeta, \boldsymbol{B} ; \boldsymbol{K}, \boldsymbol{H}]=\int[\mathrm{d} \boldsymbol{A}][\mathrm{d} \boldsymbol{c}][\mathrm{d} \overline{\boldsymbol{c}}][\mathrm{d} \boldsymbol{b}] \\
& \quad \times \exp \left[-\int \mathrm{d}^{4} x \mathcal{L}_{\ltimes}+S_{\mathrm{ext}}+S_{\mathrm{KH}}\right],
\end{aligned}
$$

where $S_{\mathrm{KH}}$ is given by

$S_{\mathrm{KH}}=\frac{1}{g^{2}} \int \mathrm{d}^{4} x[\Omega(\boldsymbol{K}, s \boldsymbol{A})-\Omega(\boldsymbol{H}, s \boldsymbol{c})]$

and $\Omega(\boldsymbol{K}, \boldsymbol{A})$ and $\Omega(\boldsymbol{H}, s \boldsymbol{c})$ read

$$
\begin{aligned}
\Omega(\boldsymbol{K}, s \boldsymbol{A}) & =\omega_{a b}\left(K_{\mathrm{T}}^{a \mu} s A_{\mathrm{T} \mu}^{b}+K_{\mathrm{T}}^{a \mu} s A_{\mathrm{Z} \mu}^{b}+K_{\mathrm{Z}}^{a \mu} s A_{\mathrm{T} \mu}^{b}\right), \\
\Omega(\boldsymbol{H}, s \boldsymbol{c}) & =\omega_{a b}\left(H_{\mathrm{T}}^{a} s c_{\mathrm{T}}^{b}+H_{\mathrm{T}}^{a} s c_{\mathrm{Z}}^{b}+H_{\mathrm{Z}}^{a} s c_{\mathrm{T}}^{b}\right) .
\end{aligned}
$$

The Feynman rules of the theory follow from $Z[\cdots]$ in (4.2) and are collected in Figs. 1, 2 and 3, where solid lines denote gauge propagators and dashed lines ghost propagators. The expressions of $D_{\mu \nu}^{a b}(p)$ and $\Delta^{a b}(p)$ in Fig. 1 are

$$
D_{\mu \nu}^{a b}(p)=g^{2} \frac{\delta^{a b}}{p^{2}}\left[\delta_{\mu \nu}+(\alpha-1) \frac{p_{\mu} p_{\nu}}{p^{2}}\right]
$$

and

$$
\Delta^{a b}(p)=-g^{2} \frac{\delta^{a b}}{p^{2}} .
$$


Fig. 1 Free propagators for $\mathrm{SU}(\mathrm{N})_{\ltimes}$ Yang-Mills in Lorenz gauge

$$
\begin{aligned}
& \stackrel{p}{\stackrel{p}{\rightarrow}} \underset{A_{\mathrm{T} \mu}^{a}(-p)}{\stackrel{b}{b}(p)}=D_{\mu \nu}^{a b}(p)
\end{aligned}
$$

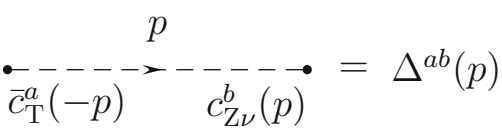

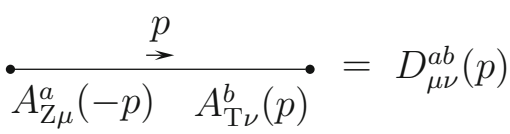

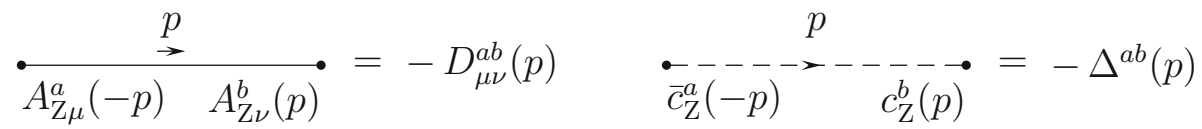

Fig. 2 Vertices for $\mathrm{SU}(\mathrm{N})_{\ltimes}$ Yang-Mills theory in Lorenz gauge
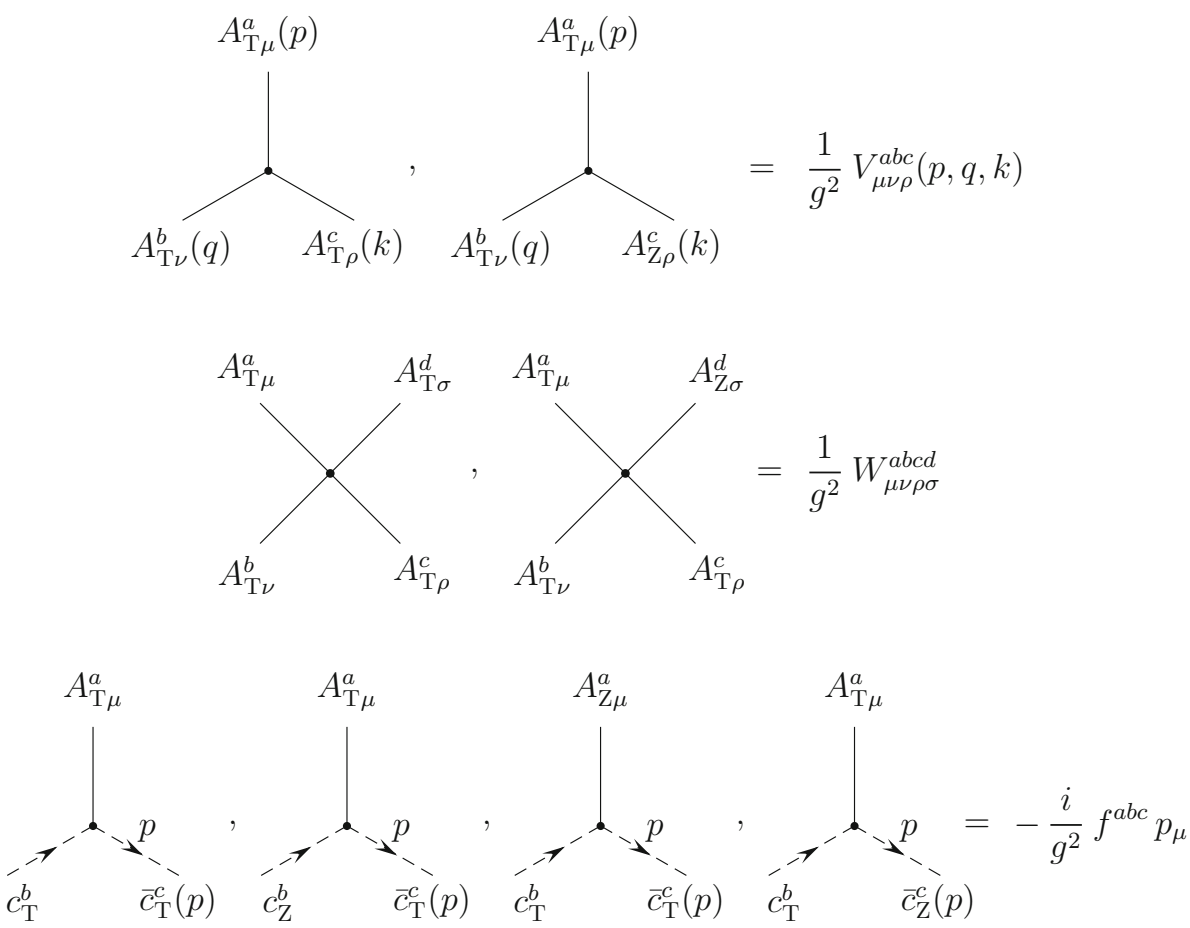

In turn, the gauge vertices $V_{\mu \nu \rho}^{a b c}(p, q, k)$ and $W_{\mu \nu \rho \sigma}^{a b c d}$ in Fig. 2 read

$$
\begin{aligned}
V_{\mu \nu \rho}^{a b c}(p, q, k)= & i f^{a b c}\left[(q-k)_{\mu} \delta_{\nu \rho}+(k-p)_{\nu} \delta_{\rho \mu}\right. \\
& \left.+(p-q)_{\rho} \delta_{\mu \nu}\right]
\end{aligned}
$$

and

$$
\begin{aligned}
W_{\mu \nu \rho \sigma}^{a b c d}= & -\left[f^{a b e} f^{c d e}\left(\delta_{\mu \rho} \delta_{\nu \sigma}-\delta_{\mu \sigma} \delta_{\nu \rho}\right)\right. \\
& +f^{a c e} f^{d b e}\left(\delta_{\mu \sigma} \delta_{\rho \nu}-\delta_{\mu \nu} \delta_{\rho \sigma}\right) \\
& \left.+f^{a d e} f^{b c e}\left(\delta_{\mu \nu} \delta_{\sigma \rho}-\delta_{\mu \rho} \delta_{\sigma \nu}\right)\right]
\end{aligned}
$$

We remark that the free propagators do not have TT components, and that their TZ component is equal to their ZT component. This will play an important role in the analysis below.

Consider for comparison conventional SU(N) Yang-Mills theory in Lorenz gauge. Its gauge fixed Lagrangian is recovered from $\mathcal{L}_{\ltimes}$ by setting all the $\mathrm{Z}$ components equal to zero. To avoid confusion, we reserve the subscripts $\mathrm{T}$ and $\mathrm{Z}$ for the field components of the $\mathrm{SU}(\mathrm{N})_{\ltimes}$ theory, and use $A_{\mu}^{a}, b^{a}, \bar{c}^{a}, c^{a}$, and $K_{\mu}^{a}, H^{a}$ without subscripts for the fields and the nonlinear BRS sources of the SU(N) theory. The gauge field and ghost free propagators of the SU(N) theory are given by $D_{\mu \nu}^{a b}(p)$ and $\Delta^{a b}(p)$ in Eqs. (4.4) and (4.5), which are equal to the $\mathrm{TZ}$ and $\mathrm{ZT}$ free propagators of the $\mathrm{SU}(\mathrm{N})_{\ltimes}$ theory. The Feynman rules for the vertices $A^{3}, A^{4}, \bar{c} A c, K A c$, and $H c c$ of the SU(N) theory are as in Figs. 2 and 3.

We now proceed to compute radiative corrections. To regulate whatever UV divergences may occur, we will use dimensional regularization with $D=4-2 \epsilon$, so from now on all diagrams and Green functions should be understood as dimensionally regularized. Since dimensional regularization manifestly preserves BRS invariance, the dimensionally regularized Green functions will solve the functional identities associated to BRS invariance. 
Fig. 3 External vertices for $\mathrm{SU}(\mathrm{N})_{\ltimes}$ Yang-Mills theory in Lorenz gauge
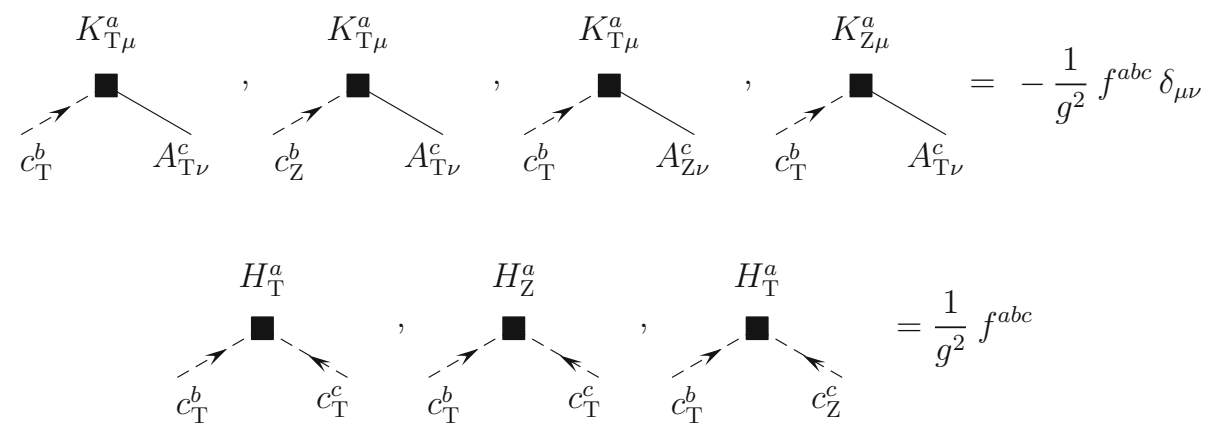

One-loop radiative corrections. The only $1 \mathrm{PI}$ one-loop diagrams that occur in perturbation theory have all their external legs of type T. To prove this, note first that the vertices of the theory, see Figs. 2 and 3, have either no or one leg of type Z. Assume now that there is a 1PI one-loop diagram with an external $\mathrm{Z}$ leg, and call $U_{1}$ to the vertex to which the leg is attached. All the other legs of $U_{1}$ will be of type T. To close a loop, two of these $\mathrm{T}$ legs must be internal. Since there are no TT propagators, each internal $\mathrm{T}$ leg must propagate into type $\mathrm{Z}$. Each one of the resulting $\mathrm{Z}$ legs will in turn be attached to a different vertex. Call these vertices $U_{2}$ and $U_{3}$. From $U_{2}$ and $U_{3}$ only $\mathrm{T}$ legs will come out. One may go on and introduce new vertices, but the loop will never close since there are no TT propagators to join two T legs. Hence 1PI one-loop diagrams have all their external legs of type $\mathrm{T}$. The only nonzero 1PI Green functions at one loop are then $\left\langle\Psi_{1 \mathrm{~T}}\left(p_{1}\right) \ldots \Psi_{n \mathrm{~T}}\left(p_{n}\right)\right\rangle_{\mathrm{SU}(N)_{\ltimes}}$, where $\Psi_{i \mathrm{~T}}$ stands for any of the fields $A_{\mathrm{T} \mu}^{a}, \bar{c}_{\mathrm{T}}^{a}, c_{\mathrm{T}}^{a}$ or the sources $K_{\mathrm{T} \mu}^{a}, H_{\mathrm{T}}^{c}$.

Consider for example the two-point 1PI function $\left\langle A_{\mathrm{T} \mu}^{a}\right.$ $\left.A_{\mathrm{T} \nu}^{b}\right\rangle_{\mathrm{SU}(\mathrm{N})_{\ltimes}}$. At one loop, it receives contributions from the diagrams in Fig. 4, where the number under each diagram is the diagram's symmetry factor. We have drawn in Fig. 5 the one-loop diagrams that contribute to the 1PI function $\left\langle A_{\mu}^{a} A_{\nu}^{b}\right\rangle_{\mathrm{SU}(\mathrm{N})}$ of SU(N) Yang-Mills theory. Since the propagators and vertices in both sets of diagrams are the same, we conclude that

$\left\langle A_{\mathrm{T} \mu}^{a} A_{\mathrm{T} \nu}^{b}\right\rangle_{\mathrm{SU}(\mathrm{N})_{\ltimes}}=2\left\langle A_{\mu}^{a} A_{\nu}^{b}\right\rangle_{\mathrm{SU}(\mathrm{N})}$.

Writing only the divergent part as $\epsilon \rightarrow 0$, this gives

$\left\langle A_{\mathrm{T} \mu}^{a}(-p) A_{\mathrm{T} \nu}^{b}(p)\right\rangle_{\mathrm{SU}(N)_{\ltimes}}=\left(\frac{13}{3}-\alpha\right) C_{\epsilon} \Pi_{\mu \nu}^{a b}(p)+O\left(\epsilon^{0}\right)$,

where $C_{\epsilon}$ is the constant

$C_{\epsilon}=-\frac{C_{2}}{16 \pi^{2} \epsilon}$.

Equation (4.6) can be extended to all 1PI functions as follows. Consider a 1PI one-loop diagram in the $\mathrm{SU}(\mathrm{N})_{\ltimes}$ theory. Since all its external legs are of type $\mathrm{T}$ and there are no TT propagators, all its vertices have one internal leg of type $\mathrm{Z}$. Label clockwise the vertices in the loop as $U_{1}, \ldots, U_{n}$. The $\mathrm{Z}$ leg coming out of vertex $U_{1}$ must be connected to an internal $\mathrm{T}$
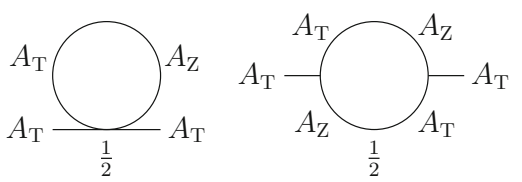<smiles>[Al]C1CCC([Al])C([Te])CCC1[Al]</smiles>

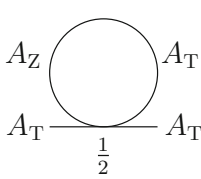

(a)

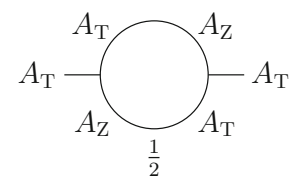

(b)

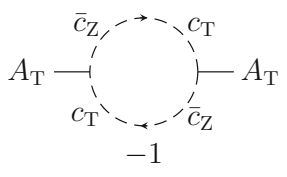

(c)
Fig. 4 One-loop corrections to $\left\langle A_{\mathrm{T}} A_{\mathrm{T}}\right\rangle_{\mathrm{SU}(\mathrm{N})_{\ltimes}}$

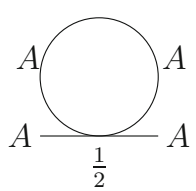

(a)

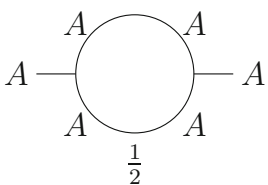

(b)<smiles>IC1CC([Tl])CCCC([Tl])C1</smiles>

(c)
Fig. 5 One-loop corrections to $\langle A A\rangle_{\mathrm{SU}(N)}$

leg of a neighboring vertex, say $U_{2}$, through a ZT propagator. In turn $U_{2}$ is connected to $U_{3}$ through another ZT propagator, and so on, until the loop is closed, with $U_{n}$ connecting to $U_{1}$ via a $\mathrm{ZT}$ propagator. For every such diagram, there is a diagram with the same vertices and the only difference that now the internal $\mathrm{Z}$ leg from $U_{1}$ connects with an internal $\mathrm{T}$ leg in $U_{n}$ through a ZT line, rather than with $U_{2}$. This implies that $U_{n}$ connects with $U_{n-1}$ through a ZT line, and so on until the loop is closed with a ZT propagator from $U_{2}$ with $U_{1}$. These two diagrams give the same contribution to the 1PI function, which in turn is equal to the contribution of the equivalent 1PI diagram in the SU(N) theory. Hence we have

$\left\langle\Phi_{1, \mathrm{~T}}\left(p_{1}\right) \ldots \Phi_{n, \mathrm{~T}}\left(p_{n}\right)\right\rangle_{\mathrm{SU}(\mathrm{N})_{\ltimes}}=2\left\langle\Phi_{1}\left(p_{1}\right) \ldots \Phi_{n}\left(p_{n}\right)\right\rangle_{\mathrm{SU}(\mathrm{N})}$.

This reduces the calculation of the one-loop 1PI Green functions in the $\mathrm{SU}(\mathrm{N})_{\ltimes}$ theory to that in the SU(N) theory. The left column In Table 1 collects all the one-loop 1PI Green functions in $\mathrm{SU}(\mathrm{N})_{\ltimes}$ Yang-Mills theory that are UV divergent, whereas the column in the center lists their UV diver- 
Table 1 UV divergent 1PI Green functions in $\mathrm{SU}(\mathrm{N})_{\ltimes}$ theory and their counterterms

\begin{tabular}{lll}
\hline 1PI UV divergent Green function & Contribution from $\bar{\Gamma}_{1}^{\epsilon}$ & Contribution from $\bar{\Gamma}_{1}^{c t}$ \\
\hline$\left\langle A_{\mathrm{T} \mu}^{a}(-p) A_{\mathrm{T} \nu}^{b}(p)\right\rangle$ & $\left(\frac{13}{3}-\alpha\right) C_{\epsilon} \Pi_{\mu \nu}^{a b}(p)$ & $-\left(c_{1}+2 c_{2}\right) \Pi_{\mu \nu}^{a b}(p)$ \\
$\left\langle A_{\mathrm{T} \mu}^{a}(p) A_{\mathrm{T} \nu}^{b}(q) A_{\mathrm{T} \rho}^{c}(k)\right\rangle$ & $\left(\frac{17}{6}-\frac{3}{2} \alpha\right) C_{\epsilon} V_{\mu \nu \rho}^{a b c}(p, q, k)$ & $-\left(c_{1}+3 c_{2}\right) V_{\mu \nu \rho}^{a b}(p, q, k)$ \\
$\left\langle A_{\mathrm{T} \mu}^{a}(p) A_{\mathrm{T} \nu}^{b}(q) A_{\mathrm{T} \rho}^{c}(k) A_{\mathrm{T} \sigma}^{d}(r)\right\rangle$ & $\left(\frac{4}{3}-2 \alpha\right) C_{\epsilon} W_{\mu \nu \rho \sigma}^{a b c d}$ & $-\left(c_{1}+4 c_{2}\right) W_{\mu \nu \rho \sigma}^{a b c d}$ \\
$\left\langle\bar{c}_{\mathrm{T}}^{a}(-p) c_{\mathrm{T}}^{b}(p)\right\rangle$ & $\frac{1}{2}(3-\alpha) C_{\epsilon} \delta^{a b} p^{2}$ & $\left(c_{2}-c_{3}\right) \delta^{a b} p^{2}$ \\
$\left\langle\bar{c}_{\mathrm{T}}^{a}(p) A_{\mathrm{T} \mu}^{b}(q) c_{\mathrm{T}}^{c}(k)\right\rangle$ & $i \alpha C_{\epsilon} f^{a b c} p_{\mu}$ & $i c_{3} f^{a b c} p_{\mu}$ \\
$\left\langle K_{\mathrm{T} \mu}^{a}(-p) c_{\mathrm{T}}^{b}(p)\right\rangle$ & $\frac{1}{2}(3-\alpha) C_{\epsilon} \delta^{a b} i p_{\mu}$ & $\left(c_{2}-c_{3}\right) \delta^{a b} i p_{\mu}$ \\
$\left\langle K_{\mathrm{T} \mu}^{a}(p) A_{\mathrm{T} \nu}^{b}(q) c_{\mathrm{T}}^{c}(k)\right\rangle$ & $-\alpha C_{\epsilon} f^{a b c} \delta_{\mu \nu}$ & $-c_{3} f^{a b c} \delta_{\mu \nu}$ \\
$\left\langle H_{\mathrm{T}}^{a}(p) c_{\mathrm{T}}^{b}(q) c_{\mathrm{T}}^{c}(k)\right\rangle$ & $-\alpha C_{\epsilon} f^{a b c}$ & $-c_{3} f^{a b c}$ \\
\hline
\end{tabular}

gent contributions as computed in dimensional regularization. The column on the right will be discussed in Sect. 5 .

Vanishing of IPI radiative corrections beyond one loop. Any 1PI $n$-loop diagram can be obtained by joining two external legs in a 1PI (n-1)-loop diagram. In our case, since 1PI one-loop diagrams have all their external legs of type $\mathrm{T}$ and there are no TT propagators, it is impossible to have two- and higher-loop 1PI diagrams.

We end this section by noting that, again because 1PI Green functions have all their external legs of type $\mathrm{T}$ and to these it is only possible to attach free TZ propagators, the only on-shell Green functions that receive radiative corrections are those having all their external legs of type $\mathrm{z}$.

\section{The BRS identity, renormalization, and unitarity}

The effective action that generates the 1PI Green functions of $G_{\ltimes}$ Yang-Mills theory is obtained by writing

$Z[\boldsymbol{J}, \bar{\zeta}, \boldsymbol{\zeta}, \boldsymbol{B} ; \boldsymbol{K}, \boldsymbol{H}]=\exp (-W[\boldsymbol{J}, \bar{\zeta}, \boldsymbol{\zeta}, \boldsymbol{B} ; \boldsymbol{K}, \boldsymbol{H}])$

and performing a Legendre transformation on $W[\boldsymbol{J}, \bar{\zeta}, \zeta$, $\boldsymbol{B} ; \boldsymbol{K}, \boldsymbol{H}]$ as follows. Introduce Legendre fields $\{\tilde{\boldsymbol{A}}, \tilde{\boldsymbol{c}}, \tilde{\overline{\boldsymbol{c}}}, \tilde{\boldsymbol{b}}\}$ for the sources $\{\boldsymbol{J}, \bar{\zeta}, \zeta, B\}$ through the functional derivatives

$$
\begin{aligned}
\tilde{A}_{\mathrm{T} \mu}^{a}(x) & =-\omega^{a b} \frac{\delta W}{\delta J_{\mathrm{Z}}^{b \mu}(x)}, \\
\tilde{A}_{\mathrm{Z} \mu}^{a}(x) & =\omega^{a b}\left[\frac{\delta W}{\delta J_{\mathrm{Z}}^{b \mu}(x)}-\frac{\delta W}{\delta J_{\mathrm{T}}^{b \mu}(x)}\right], \\
\tilde{b}_{\mathrm{T}}^{a}(x) & =-\omega^{a b} \frac{\delta W}{\delta B_{\mathrm{Z}}^{b}(x)}, \\
\tilde{b}_{\mathrm{Z}}^{a}(x) & =\omega^{a b}\left[\frac{\delta W}{\delta B_{\mathrm{Z}}^{b}(x)}-\frac{\delta W}{\delta B_{\mathrm{T}}^{b}(x)}\right], \\
\tilde{c}_{\mathrm{T}}^{a}(x) & =-\omega^{a b} \frac{\delta W}{\delta \bar{\zeta}_{\mathrm{Z}}^{b}(x)}, \\
\tilde{c}_{\mathrm{Z}}^{a}(x) & =\omega^{a b}\left[\frac{\delta W}{\delta \bar{\zeta}_{\mathrm{Z}}^{b}(x)}-\frac{\delta W}{\delta \bar{\zeta}_{\mathrm{T}}^{b}(x)}\right],
\end{aligned}
$$

$$
\begin{aligned}
\tilde{\bar{c}}_{\mathrm{T}}^{a}(x) & =\omega^{a b} \frac{\delta W}{\delta \zeta_{\mathrm{Z}}^{b}(x)}, \\
-\tilde{\bar{c}}_{\mathrm{Z}}^{a}(x) & =\omega^{a b}\left[\frac{\delta W}{\delta \zeta_{\mathrm{Z}}^{b}(x)}-\frac{\delta W}{\delta \zeta_{\mathrm{T}}^{b}(x)}\right] .
\end{aligned}
$$

Solve these equations for $\{\boldsymbol{J}, \bar{\zeta}, \zeta, \boldsymbol{B}\}$ in terms of fields $\{\tilde{\boldsymbol{A}}, \tilde{\boldsymbol{c}}, \tilde{\overline{\boldsymbol{c}}}, \tilde{\boldsymbol{b}}\}$, and use the solutions to construct the effective action functional

$$
\begin{aligned}
& \Gamma[\tilde{\boldsymbol{A}}, \tilde{\boldsymbol{c}}, \tilde{\overline{\boldsymbol{c}}}, \tilde{\boldsymbol{B}} ; \boldsymbol{K}, \boldsymbol{H}]=W[\boldsymbol{J}, \overline{\boldsymbol{\zeta}}, \zeta, \boldsymbol{B} ; \boldsymbol{K}, \boldsymbol{H}] \\
& \quad+\int \mathrm{d}^{4} x \omega_{a b}\left(J_{\mathrm{T}}^{a} \tilde{A}_{\mathrm{T}}^{b}+J_{\mathrm{T}}^{a} \tilde{A}_{\mathrm{Z}}^{b}+J_{\mathrm{Z}}^{a} \tilde{A}_{\mathrm{T}}^{b}+\bar{\zeta}_{\mathrm{T}}^{a} \tilde{c}_{\mathrm{T}}^{b}+\bar{\zeta}_{\mathrm{T}}^{a} \tilde{c}_{\mathrm{Z}}^{b}\right. \\
& \quad+\bar{\zeta}_{\mathrm{Z}}^{a} \tilde{c}_{\mathrm{T}}^{b}+B_{\mathrm{T}}^{a} \tilde{b}_{\mathrm{T}}^{b}+B_{\mathrm{T}}^{a} \tilde{b}_{\mathrm{Z}}^{b}+B_{\mathrm{Z}}^{a} \tilde{b}_{\mathrm{T}}^{b} \\
& \left.\quad+\tilde{\bar{c}}_{\mathrm{T}}^{a} \zeta_{\mathrm{T}}^{b}+\tilde{\bar{c}}_{\mathrm{T}}^{a} \zeta_{\mathrm{Z}}^{b}+\tilde{\bar{c}}_{\mathrm{Z}}^{a} \zeta_{\mathrm{T}}^{b}\right) .
\end{aligned}
$$

The very same methods as for Yang-Mills theory with semisimple gauge group show that $\Gamma$ has the form

$$
\begin{aligned}
\Gamma & =\bar{\Gamma}-\int \mathrm{d}^{4} x \omega_{a b} \\
& \times\left[\frac{\alpha}{2}\left(\tilde{b}_{\mathrm{T}}^{a} \tilde{b}_{\mathrm{T}}^{b}+2 \tilde{b}_{\mathrm{T}}^{a} \tilde{b}_{\mathrm{Z}}^{b}\right)+\tilde{b}_{\mathrm{T}}^{a} \partial \tilde{A}_{\mathrm{T}}^{b}+\tilde{b}_{\mathrm{T}}^{a} \partial \tilde{A}_{\mathrm{Z}}^{b}+\tilde{b}_{\mathrm{Z}}^{a} \partial \tilde{A}_{\mathrm{T}}^{b}\right],
\end{aligned}
$$

where the functional

$\bar{\Gamma}=\bar{\Gamma}\left[\tilde{\boldsymbol{A}}_{\mu}, \tilde{\boldsymbol{c}}, \boldsymbol{G}_{\mu}, \boldsymbol{H}\right]$

depends on $\boldsymbol{K}_{\mu}$ and $\tilde{\overline{\boldsymbol{c}}}$ through the combination

$\boldsymbol{G}_{\mu}=\boldsymbol{K}_{\mu}+\partial_{\mu} \tilde{\overline{\boldsymbol{c}}}$

and satisfies the BRS identity

$$
\begin{aligned}
\int \mathrm{d}^{4} x \omega^{a b} & {\left[\frac{\delta \bar{\Gamma}}{\delta \tilde{A}_{\mathrm{T}}^{a}} \frac{\delta \bar{\Gamma}}{\delta G_{\mathrm{Z}}^{b}}+\frac{\delta \bar{\Gamma}}{\delta \tilde{A}_{\mathrm{Z}}^{a}}\left(\frac{\delta \bar{\Gamma}}{\delta G_{\mathrm{T}}^{b}}-\frac{\delta \bar{\Gamma}}{\delta G_{\mathrm{Z}}^{b}}\right)\right.} \\
& \left.-\frac{\delta \bar{\Gamma}}{\delta \tilde{c}_{\mathrm{T}}^{a}} \frac{\delta \bar{\Gamma}}{\delta H_{\mathrm{Z}}^{b}}-\frac{\delta \bar{\Gamma}}{\delta \tilde{c}_{\mathrm{Z}}^{a}}\left(\frac{\delta \bar{\Gamma}}{\delta H_{\mathrm{T}}^{b}}-\frac{\delta \bar{\Gamma}}{\delta H_{\mathrm{Z}}^{b}}\right)\right]=0 .
\end{aligned}
$$


The analysis in Sect. 4 implies that $\bar{\Gamma}$ is the sum

$\bar{\Gamma}=\bar{\Gamma}_{0}+\hbar \bar{\Gamma}_{1}$

of a tree-level contribution

$$
\begin{aligned}
& \bar{\Gamma}_{0}=\frac{1}{g^{2}} \int \mathrm{d}^{4} x \omega_{a b}\left[\frac{1}{4} \tilde{F}_{\mathrm{T}}^{a} \tilde{F}_{\mathrm{T}}^{b}+\frac{1}{2} \tilde{F}_{\mathrm{T}}^{a} \tilde{F}_{\mathrm{Z}}^{b}-G_{\mathrm{T}}^{a} \tilde{D}_{\mathrm{T}}^{b}\right. \\
&-G_{\mathrm{T}}^{a}\left(\tilde{D} \tilde{c}_{\mathrm{Z}}^{b}+f_{c d}^{b} \tilde{A}_{\mathrm{Z}}^{c} \tilde{c}_{\mathrm{T}}^{c}\right) \\
&-G_{\mathrm{Z}}^{a} \tilde{D} \tilde{c}_{\mathrm{T}}^{b}-\frac{1}{2} f_{c d}^{b} H_{\mathrm{T}}^{a} \tilde{c}_{\mathrm{T}}^{c} \tilde{c}_{\mathrm{T}}^{d} \\
&\left.-f_{c d}^{b} H_{\mathrm{T}}^{a} \tilde{c}_{\mathrm{T}}^{c} \tilde{c}_{\mathrm{Z}}^{d}-\frac{1}{2} f_{c d}^{b} H_{\mathrm{Z}}^{a} \tilde{c}_{\mathrm{T}}^{c} \tilde{c}_{\mathrm{T}}^{d}\right]
\end{aligned}
$$

and a one-loop contribution $\bar{\Gamma}_{1}$. The term $\bar{\Gamma}_{0}$ satisfies the BRS identity (5.2). Substituting Eq. (5.3) in Eq. (5.2), it follows that $\bar{\Gamma}_{1}$ must satisfy

$\Delta \bar{\Gamma}_{1}=0$,

where $\Delta$ is the Slavnov-Taylor operator

$$
\begin{aligned}
\Delta= & \int \mathrm{d}^{4} x \omega^{a b}\left[\frac{\delta \bar{\Gamma}_{0}}{\delta \tilde{A}_{\mathrm{T}}^{a}} \frac{\delta}{\delta G_{\mathrm{Z}}^{b}}+\frac{\delta \bar{\Gamma}_{0}}{\delta G_{\mathrm{Z}}^{a}} \frac{\delta}{\delta \tilde{A}_{\mathrm{T}}^{b}}\right. \\
& +\frac{\delta \bar{\Gamma}_{0}}{\delta \tilde{A}_{\mathrm{Z}}^{a}}\left(\frac{\delta}{\delta G_{\mathrm{T}}^{b}}-\frac{\delta}{\delta G_{\mathrm{Z}}^{b}}\right)+\left(\frac{\delta \bar{\Gamma}_{0}}{\delta G_{\mathrm{T}}^{a}}-\frac{\delta \bar{\Gamma}_{0}}{\delta G_{\mathrm{Z}}^{a}}\right) \frac{\delta}{\delta \tilde{A}_{\mathrm{Z}}^{b}} \\
& -\frac{\delta \bar{\Gamma}_{0}}{\delta \tilde{c}_{\mathrm{T}}^{a}} \frac{\delta}{\delta \tilde{H}_{\mathrm{Z}}^{b}}-\frac{\delta \bar{\Gamma}_{0}}{\delta \tilde{H}_{\mathrm{Z}}^{a}} \frac{\delta}{\delta \tilde{c}_{\mathrm{T}}^{b}} \\
& \left.-\frac{\delta \bar{\Gamma}_{0}}{\delta \tilde{c}_{\mathrm{Z}}^{a}}\left(\frac{\delta}{\delta H_{\mathrm{T}}^{b}}-\frac{\delta}{\delta H_{\mathrm{Z}}^{b}}\right)-\left(\frac{\delta \bar{\Gamma}_{0}}{\delta H_{\mathrm{T}}^{a}}-\frac{\delta \bar{\Gamma}_{0}}{\delta H_{\mathrm{Z}}^{a}}\right) \frac{\delta}{\delta \tilde{c}_{\mathrm{Z}}^{b}}\right] .
\end{aligned}
$$

The very same arguments as for the semisimple case show that $\Delta$ is nilpotent, $\Delta^{2}=0$. The explicit expressions for the action of $\Delta$ on $\left(\tilde{A}_{\mathrm{T} \mu}^{a}, \tilde{A}_{\mathrm{Z} \mu}^{a}\right),\left(\tilde{c}_{\mathrm{T}}^{a}, \tilde{c}_{\mathrm{Z}}^{a}\right)\left(G_{\mathrm{T} \mu}^{a}, G_{\mathrm{Z} \mu}^{a}\right)$ and $\left(H_{\mathrm{T}}^{a}, H_{\mathrm{Z}}^{a}\right)$ are given in the appendix. The operator $\Delta$ is the quantum analog of the BRS operator and controls gauge invariance for the quantum theory. The only gauge invariant radiative corrections are those which are cohomologically nontrivial with respect to $\Delta$. That is, those that cannot be written as $\Delta X$ for any $X$. Cohomologically trivial corrections are of the form $\Delta X$, originate in gauge fixing and do not contribute to on-shell amplitudes.

We can add to $\bar{\Gamma}_{1}$ any functional $\bar{\Gamma}_{1}^{\mathrm{ct}}$ such that $\Delta \bar{\Gamma}_{1}^{\mathrm{ct}}=0$. If $\bar{\Gamma}_{1}^{\text {ct }}$ subtracts the UV divergences in $\bar{\Gamma}_{1}$, the sum $\bar{\Gamma}_{1}^{\prime}=$ $\bar{\Gamma}_{1}+\bar{\Gamma}_{1}^{\text {ct }}$ will be finite and still satisfy $\Delta \bar{\Gamma}_{1}^{\prime}=0$, thus can be taken as the one-loop contribution to the quantum effective action. Since the UV divergences in the theory are local, we are interested in the solution of equation $\Delta \bar{\Gamma}_{1}^{\mathrm{ct}}=0$ over the space of local integrated functionals of mass dimension four and ghost number zero. ${ }^{2}$ The most general solution over this space has the form

$\bar{\Gamma}_{1}^{\mathrm{ct}}=c_{1} S_{\mathrm{TT}}+\Delta X$,

where $c_{1}$ is an arbitrary real coefficient, $S_{\mathrm{TT}}$ is the G YangMills classical action

$S_{\mathrm{TT}}=\frac{1}{4} \int \mathrm{d}^{4} x \omega_{a b} \tilde{F}_{\mathrm{T}}^{a} \tilde{F}_{\mathrm{T}}^{b}$

and $X$ is any local integrated functional of mass dimension three and ghost number -1 . Note that the cohomologically nontrivial part of the solution (5.6) does not have a term

$S_{\mathrm{TZ}}=\frac{1}{2} \int \mathrm{d}^{4} x \omega_{a b} \tilde{F}_{\mathrm{T}}^{a} \tilde{F}_{\mathrm{Z}}^{b}$.

This is so since $S_{\mathrm{TZ}}$ can be written as $\Delta Y$, with $Y$ given by

$Y=\int \mathrm{d}^{4} x \omega_{a b}\left(G_{\mathrm{T}}^{a} \tilde{A}_{\mathrm{Z}}^{b}+H_{\mathrm{T}}^{a} \tilde{c}_{\mathrm{Z}}^{b}\right)$.

We observe here an important difference between the cohomologies of the BRS operator $s$ and the Slavnov-Taylor operator $\Delta$ over the space of local integrated functionals of mass dimension four and ghost number zero. While $S_{\mathrm{TT}}$ and $S_{\mathrm{TZ}}$ are both nontrivial with respect to $s$, only $S_{\mathrm{TT}}$ is nontrivial with respect to $\Delta$.

Recall now that the classical action is the sum of the terms $S_{\mathrm{TZ}}$ and $S_{\mathrm{TZ}}$. The first one of them is positive definite and the second one is not. This would seem to point to a loss of unitarity. This, however, is only apparent since, being cohomologically trivial with respect to $\Delta, S_{\mathrm{TZ}}$ does not carry gauge invariant radiative corrections in the quantum effective action.

In Sect. 4 we have used dimensional regularization to compute the one-loop contribution, call it $\bar{\Gamma}_{1}^{\text {dreg }}$, to the quantum effective action for $\mathrm{SU}(\mathrm{N})_{\ltimes}$. It consists of a divergent part $\bar{\Gamma}_{1}^{\epsilon}$ as $\epsilon \rightarrow 0$, formed by the terms listed in the left and center columns in Table 1, and a finite part $\bar{\Gamma}_{1}^{\text {fin }}$,

$\bar{\Gamma}_{1}^{\text {dreg }}=\bar{\Gamma}_{1}^{\epsilon}+\bar{\Gamma}_{1}^{\text {fin }}$.

Since dimensional regularization is BRS invariant, both $\bar{\Gamma}_{1}^{\epsilon}$ and $\bar{\Gamma}_{1}^{\text {fin }}$ satisfy $\Delta \bar{\Gamma}_{1}^{\epsilon}=\Delta \bar{\Gamma}_{1}^{\text {fin }}=0$. To remove the UV divergences, we take $\bar{\Gamma}_{1}^{\mathrm{ct}}$ as in Eq. (5.6), with $X$ given by

$X=\int \mathrm{d}^{4} x \omega_{a b}\left(c_{2} G_{\mathrm{T}}^{a} \tilde{A}_{\mathrm{T}}^{b}+c_{3} H_{\mathrm{T}}^{a} \tilde{c}_{\mathrm{T}}^{b}\right)$

and $c_{2}$ and $c_{3}$ real coefficients. The counterterm $\bar{\Gamma}_{1}^{\text {ct }}$ then produces the contributions listed in the right column of Table 1. We choose $c_{1}, c_{2}$ and $c_{3}$ so that $\bar{\Gamma}_{1}^{\epsilon}+\bar{\Gamma}_{1}^{\mathrm{ct}}=0$. This corresponds to a minimal subtraction scheme and defines a finite renormalized effective action

$\bar{\Gamma}=\lim _{\epsilon \rightarrow 0}\left(\bar{\Gamma}_{0}+\bar{\Gamma}_{1}^{\text {fin }}\right)$.

2 Both the BRS operator and the Slavnov-Taylor operator have mass dimension one and ghost number 1. 
Using the results in Table 1, we have

$c_{1}=\frac{22}{3} C_{\epsilon} \quad c_{2}=-\frac{\alpha+3}{2} C_{\epsilon}, \quad c_{3}=-\alpha C_{\epsilon}$,

with $C_{\epsilon}$ as in Eq. (4.7). These values for $c_{1}, c_{2}$ and $c_{2}$ are twice those for SU(N) Yang-Mills theory. This implies in particular that the first coefficient of the beta function for $\mathrm{SU}(\mathrm{N})_{\ltimes}$ is $-22 / 3$, rather than the usual $-11 / 3$.

Multiplicative renormalization. The subtraction performed by the counterterm $\bar{\Gamma}_{1}^{\mathrm{ct}}$ in Eqs. (5.6) and (5.10) is equivalent to multiplicative renormalization. To see this, recall that in multiplicative renormalization, the fields and the coupling constant in the tree-level action $\bar{\Gamma}_{0}$ in Eq. (5.4) are regarded as bare fields $\left\{\boldsymbol{A}_{0 \mu}, \boldsymbol{c}_{0}, \boldsymbol{G}_{0 \mu}, \boldsymbol{H}_{0}\right\}$ and bare coupling constant $g_{0}$. Renormalized quantities are then introduced through the equations

$$
\begin{aligned}
A_{0 \mathrm{~T} \mu}^{a} & =Z_{A}^{\mathrm{T}} \tilde{A}_{\mathrm{T} \mu}^{a}, & A_{0 \mathrm{Z} \mu}^{a} & =Z_{A}^{\mathrm{Z}} \tilde{A}_{\mathrm{Z} \mu}^{a}, \\
c_{0 \mathrm{~T}}^{a} & =Z_{c}^{\mathrm{T}} \tilde{c}_{\mathrm{T}}^{a}, & c_{0 \mathrm{Z}}^{a} & =Z_{c}^{\mathrm{Z}} \tilde{c}_{\mathrm{Z}}^{a}, \\
G_{0 \mathrm{~T}}^{a} & =Z_{G}^{\mathrm{T}} G_{\mathrm{T}}^{a}, & G_{0 \mathrm{Z}}^{a} & =Z_{G}^{\mathrm{Z}} G_{\mathrm{Z} \mu}^{a}, \\
H_{0 \mathrm{~T}}^{a} & =Z_{G}^{\mathrm{T}} H_{\mathrm{T}}^{a}, & H_{0 \mathrm{Z}}^{a} & =Z_{G}^{\mathrm{Z}} H_{\mathrm{Z}}^{a}
\end{aligned}
$$

and

$g_{0}=Z_{g} g$.

Writing every renormalization constant as $Z=1+\delta Z$, with $\delta Z$ first-order in perturbation theory, the action $\bar{\Gamma}_{0}\left[\Psi_{0}, g_{0}\right]$ is recast as

$$
\begin{aligned}
\bar{\Gamma}_{0}\left[\tilde{\boldsymbol{A}}_{0}, \tilde{\boldsymbol{c}}_{0}, \boldsymbol{G}_{0}, \boldsymbol{H}_{0}, g_{0}\right] & =\bar{\Gamma}_{0}[\tilde{\boldsymbol{A}}, \tilde{\boldsymbol{c}}, \boldsymbol{G}, \boldsymbol{H}, g] \\
& +\delta \bar{\Gamma}_{0}[\tilde{\boldsymbol{A}}, \tilde{\boldsymbol{c}}, \boldsymbol{G}, \boldsymbol{H}, g],
\end{aligned}
$$

where the counterterm $\delta \bar{\Gamma}_{0}[\cdots]$ collects all contributions of order one,

$$
\begin{aligned}
& \delta \bar{\Gamma}_{0}[\tilde{\boldsymbol{A}}, \tilde{\boldsymbol{c}}, \boldsymbol{G}, \boldsymbol{H}, g]=\frac{1}{g^{2}} \int \mathrm{d}^{4} x \omega_{a b} \\
& \quad \times\left[\frac{1}{2}\left(2 \delta Z_{A}^{\mathrm{T}}-2 \delta Z_{g}\right) \tilde{A}_{\mathrm{T}}^{a \mu}\left(\delta_{\mu \nu} \partial^{2}-\partial_{\mu} \partial_{\nu}\right) \tilde{A}_{\mathrm{T}}^{b v}+\cdots\right] .
\end{aligned}
$$

The requirement that $\delta \bar{\Gamma}_{0}[\tilde{\boldsymbol{\Psi}}, g]$ must cancel the UV divergences fixes

$$
\begin{aligned}
\delta Z_{g} & =-\frac{g^{2} c_{1}}{2}, \quad \delta Z_{c}^{\mathrm{T}}+\delta Z_{G}^{\mathrm{T}}=-g^{2}\left(c_{1}+c_{2}+c_{3}\right), \\
\delta Z_{A}^{\mathrm{T}}=g^{2} c_{2}, & \delta Z_{H}^{\mathrm{T}}+2 \delta Z_{c}^{\mathrm{T}}=g^{2}\left(c_{3}-c_{1}\right),
\end{aligned}
$$

where $c_{1}, c_{2}$, and $c_{3}$ are the coefficients in the right column in Table 1. Since there are no one-loop 1PI diagrams with $\mathrm{Z}$ external legs, there are no conditions for $\delta Z_{\Psi}^{Z}$. The question then arises as to what is the meaning of the $\mathrm{Z}$ terms in $\delta \bar{\Gamma}_{0}[\tilde{\boldsymbol{\Psi}}, g]$. Our analysis above indicates that they should be cohomologically trivial with respect to the Slavnov-Taylor operator. This is indeed the case. A long but straightforward calculation shows that, for

$\delta Z_{A}^{Z}=\delta Z_{A}^{\mathrm{T}}, \quad \delta Z_{c}^{\mathrm{Z}}=\delta Z_{c}^{\mathrm{T}}, \quad \delta Z_{G}^{\mathrm{Z}}=\delta Z_{G}^{\mathrm{T}}, \quad \delta Z_{H}^{\mathrm{Z}}=\delta Z_{H}^{\mathrm{T}}$, the functional $\delta \bar{\Gamma}_{0}[\tilde{\boldsymbol{A}}, \tilde{\boldsymbol{c}}, \boldsymbol{G}, \boldsymbol{H}, g]$ can be written as

$\delta \bar{\Gamma}_{0}[\tilde{\boldsymbol{A}}, \tilde{\boldsymbol{c}}, \boldsymbol{G}, \boldsymbol{H}, g]=c_{1} S_{\mathrm{TT}}+\Delta(X+U)$,

where $X$ is given by Eq. (5.10) and $U$ has the form

$$
\begin{aligned}
U= & \int \mathrm{d}^{4} x \omega_{a b}\left[\left(c_{1}+c_{2}\right) G_{\mathrm{T}}^{a} \tilde{A}_{\mathrm{Z}}^{b}-\left(c_{1}+2 c_{2}+c_{3}\right) H_{\mathrm{T}}^{a} \tilde{c}_{\mathrm{Z}}^{b}\right. \\
& \left.+c_{2} G_{\mathrm{Z}}^{a} \tilde{A}_{\mathrm{T}}^{b}-c_{3} H_{\mathrm{Z}}^{a} \tilde{c}_{\mathrm{T}}^{b}\right] .
\end{aligned}
$$

The difference between the counterterms $\delta \bar{\Gamma}_{0}$ above and $\bar{\Gamma}_{1}^{\mathrm{ct}}$ in Eqs. (5.6) and (5.10) is $\Delta U$, which is cohomologically trivial.

All in all, the only gauge invariant radiative corrections are those in $c_{1}$, which account for a renormalization of the coupling constant. This introduces a renormalization scale in the quantum effective action and the quantum theory is asymptotically free.

\section{Discussion}

The pattern observed for the gauge invariant degrees of freedom in the quantum theory resembles very much that for the self-antiself dual instantons of the classical theory [16]. In the classical case, the number of collective coordinates of the $G_{\ltimes}$ instantons is twice that of the embedded $G$ instantons, yet $\omega_{a b} F_{\mathrm{T} \mu \nu}^{a} F_{\mathrm{Z}}^{b \mu \nu}$ does not contribute to the instanton number. Now the gauge invariant radiative corrections are doubled and $\omega_{a b} F_{\mathrm{T} \mu \nu}^{a} F_{\mathrm{Z}}^{b \mu \nu}$ is cohomologically trivial with respect to the Slavnov-Taylor operator.

Our discussion may have some implications for YangMills theories with more general nonreductive real metric Lie algebras. There is a structure theorem [17] that states that all real metric Lie algebras are direct products of Abelian algebras, simple real Lie algebras, and double extensions $\mathfrak{d}(\mathfrak{h}, \mathfrak{g})$ of a real metric Lie algebra $\mathfrak{h}$ by an algebra $\mathfrak{g} .{ }^{3}$ The double extension $\mathfrak{d}(\mathfrak{h}, \mathfrak{g})$ is obtained $[14,17]$ by forming the classical double $\mathfrak{g}_{\ltimes}$ and then by acting with $\mathfrak{g}$ on $\mathfrak{h}$ via antisymmetric derivations. Incidentally we mention that the classical double $\mathfrak{g}_{\ltimes}$ can be viewed as the double extension of the trivial algebra by $\mathfrak{g}$.

According to the theorem, since $\mathfrak{h}$ must be metric, three possibilities must be considered for $\mathfrak{h}$ in forming double extensions $\mathfrak{d}(\mathfrak{h}, \mathfrak{g})$. The first one is that $\mathfrak{h}$ is a simple real Lie algebra. In this case [14], the algebra of antisymmetric derivations of $\mathfrak{h}$ is $\mathfrak{h}$ itself and the double extension $\mathfrak{d}(\mathfrak{h}, \mathfrak{h})$ is isomorphic to the direct product $\mathfrak{h} \times \mathfrak{h}_{\ltimes}$. The resulting

\footnotetext{
3 The theorem goes further and specifies the nature of $\mathfrak{g}$ in the double extension.
} 
Yang-Mills theory then separates into two Yang-Mills theories, not interacting with each other, one with gauge group $H$ and one with group $H_{\ltimes}$. The second possibility is that $\mathfrak{h}$ is Abelian, of dimension $m$. Being Abelian, any nondegenerate, symmetric bilinear form on $\mathfrak{h}$ is a metric, and it can always be brought to a diagonal form with all the entries in the diagonal equal to +1 and -1 . If the number of occurrences of +1 is $p$, and the number of occurrences of -1 is $q$, the algebra of antisymmetric derivations of $\mathfrak{h}$ is any subalgebra of $\mathfrak{s o}(p, q)$ [14]. Many of the nonsemisimple WZW models considered in the literature [8-13] and their four-dimensional Yang-Mills analogs [15] fall into this class. In this instance unitarity remains an open problem. We think that a thorough analysis of the corresponding Slavnov-Taylor operator should shed some light on the problem. The third possibility for $\mathfrak{h}$ is that it is a double extension, which takes us back to the starting point.

Acknowledgments This work was partially funded by the Spanish Ministry of Economy and Competitiveness through Grant FPA201454154-P and by the European Union Cost Program through Grant MP 1405.

Open Access This article is distributed under the terms of the Creative Commons Attribution 4.0 International License (http://creativecomm ons.org/licenses/by/4.0/), which permits unrestricted use, distribution, and reproduction in any medium, provided you give appropriate credit to the original author(s) and the source, provide a link to the Creative Commons license, and indicate if changes were made.

Funded by SCOAP ${ }^{3}$.

\section{Appendix}

The action of the Slavnov-Taylor operator (5.5) on the fields $\left(\tilde{A}_{\mathrm{T} \mu}^{a}, \tilde{A}_{\mathrm{Z} \mu}^{a}\right)$ and $\left(\tilde{c}_{\mathrm{T}}^{a}, \tilde{c}_{\mathrm{Z}}^{a}\right)$ is given by

$$
\begin{aligned}
& \Delta \tilde{A}_{\mathrm{T} \mu}^{a}=-\tilde{D}_{\mu} \tilde{c}_{\mathrm{T}}^{a}, \\
& \Delta \tilde{A}_{\mathrm{Z} \mu}^{a}=-\left(\tilde{D}_{\mu} \tilde{c}_{\mathrm{T}}^{a}+f_{b c}{ }^{a} \tilde{A}_{\mathrm{Z} \mu}^{b} \tilde{c}_{\mathrm{T}}^{c}\right), \\
& \Delta \tilde{c}_{\mathrm{T}}^{a}=\frac{1}{2} f_{b c}{ }^{a} \tilde{c}_{\mathrm{T}}^{b} \tilde{c}_{\mathrm{T}}^{c}, \\
& \Delta \tilde{c}_{\mathrm{Z}}^{a}=f_{b c}{ }^{a} \tilde{c}_{\mathrm{T}}^{b} \tilde{c}_{\mathrm{Z}}^{c},
\end{aligned}
$$

Modulo an irrelevant overall sign, these expressions generalize the classical BRS operator $s$ in Eqs. (3.9) and (3.10). The action on $\left(G_{\mathrm{T} \mu}^{a}, G_{\mathrm{Z} \mu}^{a}\right)$ and $\left(H_{\mathrm{T}}^{a}, H_{\mathrm{Z}}^{a}\right)$ is in turn

$$
\begin{aligned}
\Delta G_{\mathrm{T} \mu}^{a}= & -\left(\tilde{D}^{\rho} \tilde{F}_{\mathrm{T} \rho \mu}^{a}+f^{a b c} G_{\mathrm{T} \mu}^{b} \tilde{c}_{\mathrm{T}}^{c}\right), \\
\Delta G_{\mathrm{Z} \mu}^{a}= & -\left(\tilde{D}^{\rho} \tilde{F}_{\mathrm{Z} \rho \mu}^{a}+f_{b c}{ }^{a} \tilde{A}_{\mathrm{Z}}^{b \rho} \tilde{F}_{\mathrm{T} \rho \mu}^{c}\right) \\
& +f_{b c}{ }^{a}\left(G_{\mathrm{Z} \mu}^{b} \tilde{c}_{\mathrm{T}}^{c}+G_{\mathrm{T} \mu}^{b} \tilde{c}_{\mathrm{Z}}^{c}\right),
\end{aligned}
$$

$$
\begin{aligned}
& \Delta H_{\mathrm{T}}^{a}=\tilde{D}_{\mu} G_{\mathrm{T}}^{a \mu}-f_{b c}{ }^{a} H_{\mathrm{T}}^{b} \tilde{c}_{\mathrm{T}}^{c}, \\
& \Delta H_{\mathrm{Z}}^{a}=\tilde{D}_{\mu} G_{\mathrm{Z}}^{a \mu}-f_{b c}{ }^{a} G_{\mathrm{T} \mu}^{b \mu} \tilde{A}_{\mathrm{Z}}^{c \mu}-f_{b c}{ }^{a}\left(H_{\mathrm{Z}}^{b} \tilde{c}_{\mathrm{T}}^{c}+H_{\mathrm{T}}^{b} \tilde{c}_{\mathrm{Z}}^{c}\right) .
\end{aligned}
$$

With this, it is matter of algebra to check that (i) the action of $\Delta$ on $\bar{\Gamma}_{1}^{\mathrm{ct}}$ in Eqs. (5.6) and (5.10) produces the terms in the right column of Table 1, (ii) that $S_{\mathrm{TZ}}$ in Eq. (5.8) equals $\Delta Y$, with $Y$ given by Eq. (5.9), and (iii) that $\delta \bar{\Gamma}_{0}[\tilde{\boldsymbol{A}}, \tilde{\boldsymbol{c}}, \boldsymbol{G}, \boldsymbol{H}, g]$ can be written as in Eq. (5.12).

\section{References}

1. A. Achúcarro, P.K. Townsend, A Chern-Simons action for threedimensional anti-de Sitter supergravity theories. Phys. Lett. B 180, 89 (1986)

2. E. Witten, (2+1)-Dimensional Gravity as an Exactly Soluble System. Nucl. Phys. B 311, 46 (1988)

3. G. Barnich, B. Oblak, Notes on the BMS group in three dimensions: I. Induced representations. JHEP 1406, 129 (2014). arXiv:1403.5803 [hep-th]

4. G. Barnich, B. Oblak, Notes on the BMS group in three dimensions: II. Coadjoint representation, JHEP 1503, 033 (2015). arXiv: 1502.00010 [hep-th]

5. G. Barnich, C. Troessaert, Symmetries of asymptotically flat 4 dimensional spacetimes at null infinity revisited. Phys. Rev. Lett. 105, 111103 (2010). arXiv:0909.2617 [gr-qc]

6. M. Hatsuda, K. Kamimura, W. Siegel, Type II chiral affine Lie algebras and string actions in doubled space. JHEP 1509, 113 (2015). arXiv:1507.03061 [hep-th]

7. R.F. Dashen, E.E. Jenkins, A.V. Manohar, The 1, N(c) expansion for baryons, Phys. Rev. D 49, 4713, erratum Phys. Rev. D 51(1995), 2489 (1994). arXiv:hep-ph/9310379

8. C.R. Nappi, E. Witten, A WZW model based on a nonsemisimple group. Phys. Rev. Lett. 71, 3751 (1993). arXiv:hep-th/9310112

9. K. Sfetsos, Exact string backgrounds from WZW models based on nonsemisimple groups. Int. J. Mod. Phys. A 9, 4759 (1994). arXiv:hep-th/9311093

10. K. Sfetsos, Gauging a nonsemisimple WZW model. Phys. Lett. B 324, 335 (1994). arXiv:hep-th/9311010

11. K. Sfetsos, Gauged WZW models and nonAbelian duality. Phys. Rev. D 50, 2784 (1994). arXiv:hep-th/9402031

12. N. Mohammedi, On bosonic and supersymmetric current algebras for nonsemisimple groups. Phys. Lett. B 325, 371 (1994). arXiv:hep-th/9312182

13. J.M. Figueroa-O'Farrill, S. Stanciu, Nonreductive WZW models and their CFTs. Nucl. Phys. B 458, 137 (1996). arXiv:hep-th/9506151

14. J.M. Figueroa-O'Farrill, S. Stanciu, Nonsemisimple Sugawara constructions. Phys. Lett. B 327, 40 (1994). arXiv:hep-th/9402035

15. A.A. Tseytlin, On gauge theories for nonsemisimple groups. Nucl. Phys. B 450, 231 (1995). arXiv:hep-th/9505129

16. F. Ruiz Ruiz, YangMills theory for semidirect products $G \ltimes \mathfrak{g}^{*}$ and its instantons. Eur. Phys. J. C 75, 317 (2015). arXiv: 1408.1049 [hep-th]

17. A. Medina, $\mathrm{Ph}$ Revoy, Algèbres de Lie et produit scalaire invariant. Ann. Scient. Éc. Norm. Sup. 18, 553 (1985) 